ИЗВЕСТИЯ АҚАДЕМИИ НАУК ЭСТОНСКОИ ССР. ТОМ ХІV СЕРИЯ ФИЗИКО-МАТЕМАТИЧЕСКИХ И ТЕХНИЧЕСКИХ НАУК. 1965, № 2

\title{
ПОКАЗАТЕЛЬ ПЕРЕГРУЗКИ И КОНДЕНСАЦИЯ В ПРЕПАРАТИВНОЙ ГАЗОВОЙ ХРОМАТОГРАФИИ
}

В газовой хроматографии колонки характеризуются в основном критериями разделения [1]. В препаративных работах полезными характеристиками являются, кроме того, производительность $[2,3]$ колонки и выход конденсата.

Так как производительность и частично выход конденсата зависят от однократного количества пробы, вводимой в колонку, то в препаративной хроматографии работают главным образом в диапазоне перегрузки. При этом следует, однако, отметить, что до сих пор отсутствует удобный количественный показатель перегрузки колонки. Часто используемая характеристика - максимальное однократное количество разделяемого вещества остается неточным, если одновременно не указать отношений удерживаемых объемов и критериев разделения. В этом смысле лучше характеризует колонку зависимость высоты теоретической тарелки от нагрузки $\left[{ }^{4}\right]$.

Мы считали целесообразным связать показатель перегрузки колонки с формой хроматографического пика, так как от формы пика зависит выход конденсата.

Будем считать, что в узком диапазоне увеличения нагрузки колонки действительно уравнение

$$
\frac{H_{1}^{g-1}}{b_{1}}=\frac{H_{2}^{g-1}}{b_{2}},
$$

где $H$ - высота и $b-$ ширина пика.

Показатель перегрузки $g$ стражает характер расширения пика при увеличении нагрузки $V=\frac{1}{2} b H$. При малых нагрузках $g \approx 1$ и пик при увеличении $V$ не расширяется. В первой стадии перегрузки $g \approx 2$ и наряду с увеличением высоты пика $H$ пропорционально увеличивается и ширина его $b$. При сильной перегрузке $g \rightarrow \infty$. Практически $g$ не превышает 3. При этом значении $g$ наблюдается сильное расширение пика. Для препаративной работы можно рекомендовать диапазон $1,2<g<$ $<1,6$. Как показывают опыты, раз-
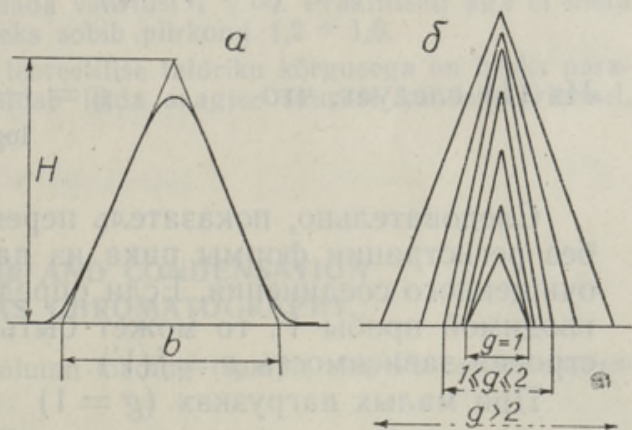

Рис. 1. Хроматографический пик: $a-$ размеры пика; 6 - изменение формы пика при увеличении нагрузки. личные количества разных соединений могут вызывать одну и ту же перегрузку.

Из разделенного компонента конденсируется часть, концентрация которого в газе-носителе превышает концентрацию насыщенных паров того же компонента при температуре конденсации [5]. 


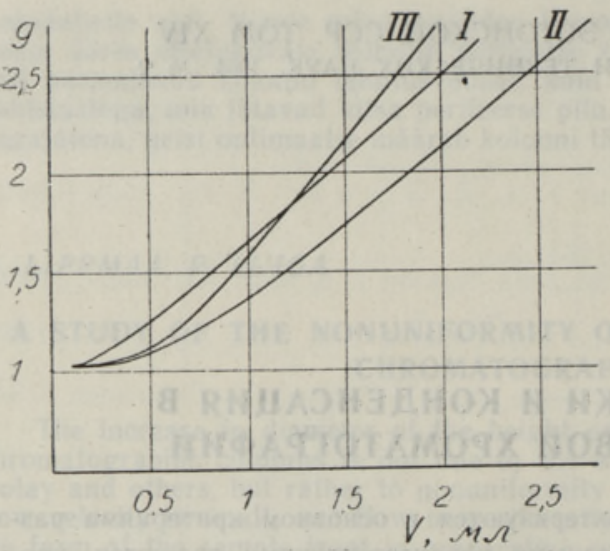

Рис. 2. Зависимость показателя перегрузки $g$ от нагрузки $V$. Колонка: диаметр 32 мм, длина 3,2 , динатомитовыі кирпич $0,25-0,5$ мм, пропитанный $30 \%$ дибутилфталатом; I - диэтиловый эфир, II - $\mu$-гексан, III - $\mu$-гептан.

\section{Выход выражается формулой}

$$
p=\frac{K}{V} \text {. }
$$

Максимальное количество пробы, которое при данных условиях конденсации еще не дает конденсата, показано на рис. 3 (площадь $M$ ). Хотя гоказатель перегрузки $g$ является плавной функцией от количества вводимой пробы $V$, в узком диапазоне величины $V$ его можно считать постоянным. Соответственно, согласно уравнению (1).

откуда

$$
\frac{H_{V}^{g-1}}{b_{V}}=\frac{H_{M}^{g-1}}{b_{M}},
$$

$$
H_{M} b_{M}=H_{V} b_{V}\left(1-\frac{H_{V}-H_{M}}{H_{V}}\right)^{g} \text {. }
$$

Так как $\quad H_{M} b_{M}=2 M, \quad H_{V} b_{V}=2 V \quad$ и $\quad\left(\frac{H_{V}-H_{M}}{H_{V}}\right)^{2}=p$.

$$
\text { Можно написать } \quad M=V(1-\sqrt{p})^{g}
$$

Рис. 3. Определение выхода конденсата: $c$ - концентрация насыщенных паров вещества при температуре конденсации; $K$ - конденсируемая часть пробы; $M-$ минимальное количество пробы, необходимое для получения конденсата.

или для вычисления ожидаемого выхода при конденсации

$$
p=\left(1-\sqrt[g]{\frac{M}{V}}\right)^{2}
$$



Из (1) следует, что

$$
g=\frac{\log \frac{V_{2}}{V_{1}}}{\log \frac{1-\sqrt{p_{1}}}{1-\sqrt{p_{2}}}}
$$

Следовательно, показатель перегрузки $g$ может быть определен даже без регистрации формы пика из данных о выходе $p$ хроматографически очищенного соединения. Если определить выход $p$ для малого количества вводимой пробы $V$, то может быть вычислено $M$, а из этих данных построена зависимость $p=f(V)$.

При малых нагрузках $(g=1)$

$$
p=\frac{(V-M)^{2}}{V^{2}} \text {. }
$$

При малых значениях $g$ из выражения (5) получаем приблизительно

$$
g=\left(\frac{b_{2}}{b_{1}}\right)^{\frac{1}{\ln \frac{V_{2}}{V_{1}}}}=\left(\frac{n_{1}}{n_{2}}\right)^{\frac{1}{2 \ln \frac{V_{2}}{V_{1}}}},
$$

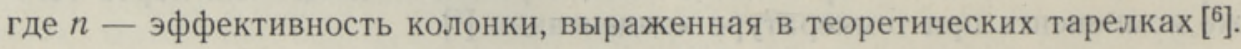


Из выражения (7) следует, что $g^{2}$ приблизительно показывает, во сколько раз падает эффективность колонки при увеличении вводимой пробы в $e=2,718$ раза.

\section{Выводы}

1. Разработан способ определения показателя перегрузки колонки $(g)$, отражающего характер расширения пика при увеличении нагрузки хроматографической колонки.

2. Предложена методика установления зависимости выхода конденсата очищенного соединения от условий опыта.

\section{ЛИТЕРАТУ РА}

‥ Ж уховицки й А. А., Т уркельт ауб Н. М., Газовая хроматография, Гостоптехиздат, М., 1962.

2. Sakodynski K. I., Schulz H., Schomburg C., Gas chromatography 1962, Ed. M. van Swany, Butterworths, Washington, 1962, p. 64.

3. Алексеева К. В., Жуховицкий А. А., Туркельтауб Н. М., Нефтехимия, 2, 934 (1962).

4. B a y e r E., W it s ch H. G., Z. Anal. Chem., 181, 384 (1961).

5. Э й зен О. Г., Луй га П. О., Газовая хроматография (Тр. II Всесоюзн. конф.). «Наука», М., 1964, стр. 216.

б. К ейлем а н с А., Хроматография газов, Изд. иностр. лит., М., 1959.

Ннститут кибернетики

Академии наук Эстонской ССР
Поступила в редакцию 29/VI 1964

\section{P. LUIGA, E. LIPPMAA}

\section{KOORMATUSE NÄITAJA JA KONDENSATSIOON PREPARATIIVSES GAASIKROMATOGRAAFIAS}

Piigi kuju muutust, olenevalt kolonni koormatusest (proovi kogus $V$ ), saab analüütiJiselt văljendada seosega

$$
\frac{H_{1}^{g-1}}{b_{1}}=\frac{H_{2}^{g-1}}{b_{2}},
$$

kus $H$ on piigi kōrgus, $b$ - piigi aluse laius ja $g$ - koormatuse näitaja. $g$ on muutuv suurus, sõltudes proovi kogusest, ja võib omada väärtusi $1 \div \infty$. Praktiliselt aga ei ületa g väärtus 3 ja preparatiivseteks lahutamisteks sobib piirkond $1,2 \div 1,6$.

Proovi kogusele vastav $g$ väärtus koos teoreetilise taldriku körgusega on heaks parameetriks preparatiivsele kolonnile ja võimaldab leida saagise suurust kondenseerimisel, :sōltuvalt proovi kogusest.

\section{P. LUIGA, E. LIPPMAA}

\section{THE LOADING FACTOR AND CONDENSATION IN LARGE-SCALE GAS CHROMATOGRAPHY}

The dependence of peak shape on the column loading (sample size $V$ ) can be expressed analytically as

$$
\frac{H_{1}{ }^{g-1}}{b_{1}}=\frac{H_{2}{ }^{g-1}}{b_{2}}
$$

where $H$ is peak height, $b$ is peak width at base and $g$ is loading factor. The loading Tactor $g$ depends on sample size and can assume values from 1 to $\infty$, yet in practice does not exceed 3 with values from 1.2 to 1.6 recommended for preparative separations,

The value of $g$ for a particular sample size is, together with the equivalent plate height, a useful parameter of preparative columns and can be used for finding the dependsence of the condensation efficiency on sample size.

6 ENSV TA Toimetised F-2 65. 